\title{
Reviewing model calculations of the Collins fragmentation function
}

\author{
Daniela Amrath, ${ }^{1,2, *}$ Alessandro Bacchetta, ${ }^{3, \dagger}$ and Andreas Metz ${ }^{1, \ddagger}$ \\ ${ }^{1}$ Institut für Theoretische Physik II, Ruhr-Universität Bochum, D-44780 Bochum, Germany \\ ${ }^{2}$ Deutsches Elektronen-Synchroton DESY, D-22603 Hamburg, Germany \\ ${ }^{3}$ Institut für Theoretische Physik, Universität Regensburg, D-93040 Regensburg, Germany
}

(Received 21 April 2005; published 30 June 2005)

The Collins fragmentation function describes a left/right asymmetry in the fragmentation of a transversely polarized quark into a hadron in a jet. Four different model calculations of the Collins function have been presented in the literature. While based on the same concepts, they lead to different results and, in particular, to different signs for the Collins function. The purpose of the present work is to review the features of these models and correct some errors made in previous calculations. A full study of the parameter dependence and the possible modifications to these models is beyond the scope of the paper. However, some general conclusions are drawn.

DOI: 10.1103/PhysRevD.71.114018

PACS numbers: 13.60.Le, 13.87.Fh, 12.39.Fe

\section{INTRODUCTION}

The Collins fragmentation function [1] contributes to several single-spin asymmetries in hard scattering reactions, e.g. semi-inclusive deep inelastic scattering (DIS) [2,3], proton-proton collisions [4], and electron-positron annihilation into hadrons [5]. Several phenomenological works on the Collins function have been published in the past years (see, e.g., Refs. [6-10]), based on experimental data coming from the above-mentioned processes [11-15]. In particular, the HERMES collaboration has recently extracted a component of the transverse spin asymmetry in semi-inclusive DIS [16], providing up to now the cleanest evidence of the existence of a nonzero Collins function. Although the interpretation of the data is still under debate-because of the unexpected relative behavior of $\pi^{+}$, $\pi^{0}$, and $\pi^{-}$asymmetries - the favored Collins function for pions seems to be positive and small [17]. The COMPASS collaboration performed the same kind of measurement using a deuteron target, obtaining asymmetries consistent with zero [18]. This could be due simply to the difference between the targets.

Four model calculations of the Collins function for the fragmentation of a quark into a pion have been presented so far in the literature [19-22] and used to make predictions and/or compare to available data [21,23-25]. All of them produce the necessary imaginary parts by adopting a simple model for the fragmentation process at tree level and inserting one-loop corrections. Two possibilities have been investigated for the tree-level amplitudes and two possible

\footnotetext{
*Electronic address: daniela.amrath@desy.de

${ }^{\dagger}$ Electronic address: alessandro.bacchetta@physik.uniregensburg.de

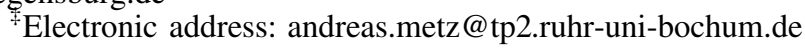

kinds of one-loop corrections, for a total of four different models: pseudoscalar pion-quark coupling with pion loops [19] and with gluon loops [21]; pseudovector pion-quark coupling with pion loops [20] and with gluon loops [22]. We discovered an overall sign error in the Collins function calculated in Refs. [19,20,22], and a more fundamental error in Ref. [21], as we are going to discuss in the paper.

The calculation in Ref. [21] makes also use of Gaussian form factors at the pion-quark vertex. In the present analysis, we refrain ourselves from taking into account different types of form factors and we limit ourselves to showing the features of the models when using pointlike vertices. It would be interesting to study how our results change upon the introduction of form factors of different types, which is however beyond the scope of the present article. Moreover, such models are purely phenomenological and cannot be derived from a Lagrangian of a microscopic model.

The paper is organized as follows: in Sec. II we use a pseudoscalar pion-quark coupling to calculate the unpolarized fragmentation function $D_{1}$, as well as the Collins fragmentation function generated by pion and gluon loops. We also outline the general ideas underlying the application of the various models to the calculation of fragmentation functions. In Sec. III we repeat the same calculations using a pseudovector pion-quark coupling. In Sec. IV we discuss numerical results obtained with both versions of the pion-quark coupling and, in particular, we give some estimates of the Collins single transverse spin asymmetry in semi-inclusive DIS.

\section{PSEUDOSCALAR PION-QUARK COUPLING}

Fragmentation functions can be calculated from the correlation function $\Delta\left(z, \vec{k}_{T}\right)$ [26], 


$$
\begin{aligned}
& \Delta\left(z, \vec{k}_{T}\right)=\left.\frac{1}{4 z} \int d k^{+} \Delta(k, p)\right|_{k^{-}=p^{-} / z} \\
& =\sum_{X} \int \frac{d \xi^{+} d^{2} \vec{\xi}_{T}}{4 z(2 \pi)^{3}} e^{+i k \cdot \xi}\left\langle 0\left|\mathcal{U}^{T}\left[\infty_{T}, \vec{\xi}_{T} ;-\infty^{+}\right] \mathcal{U}^{+}\left[-\infty^{+}, \xi^{+} ; \vec{\xi}_{T}\right] \psi(\xi)\right| \pi, X\right\rangle \\
& \times\left.\left\langle\pi, X\left|\bar{\psi}(0) \mathcal{U}^{+}\left[0^{+},-\infty^{+} ; 0_{T}\right] \mathcal{U}^{T}\left[0_{T}, \infty_{T} ;-\infty^{+}\right]\right| 0\right\rangle\right|_{\xi^{-}=0} .
\end{aligned}
$$

Since the two quark fields $\psi(0)$ and $\psi(\xi)$ transform differently under color gauge transformations a gauge link is included in order to ensure color gauge invariance of the correlator. The notation $\mathcal{U}^{+}\left[a^{+}, b^{+} ; c_{T}\right]$ indicates a gauge link running along the plus direction from $\left(0^{-}, a^{+}, c_{T}\right)$ to $\left(0^{-}, b^{+}, c_{T}\right)$, while $\mathcal{U}^{T}\left[a_{T}, b_{T} ; c^{+}\right]$indicates a gauge link running along the transverse direction from $\left(0^{-}, c^{+}, a_{T}\right)$ to $\left(0^{-}, c^{+}, b_{T}\right)$. The specific path of the link connecting the quark fields follows from the derivation of QCD factorization. The definition written above applies to the correlation function appearing in semi-inclusive DIS, while in $e^{+} e^{-}$ annihilation all occurrences of $-\infty^{+}$in the gauge links should be replaced by $\infty^{+}$. However, in Ref. [27] it was shown that by means of a certain contour deformation one can derive factorization in such a way that both the fragmentation functions in semi-inclusive DIS and in $e^{+} e^{-}$ annihilation have future-pointing gauge links. This universality of fragmentation functions was also observed earlier in the context of a specific model calculation [28].

It is convenient to evaluate the correlator in Eq. (1) in Feynman gauge, which we shall utilize in the rest of the article. In Feynman gauge one has only to consider those pieces of the link that run along the light-cone while the transverse gauge links $\mathcal{U}^{T}$ give no contribution and can be neglected [29,30].

The tree-level diagram describing the fragmentation of a virtual (timelike) quark into a pion is depicted in Fig. 1. In the models used here the final state $|\pi X\rangle$ is described by the detected pion and a (unobserved) quark. Once higher order corrections are included the quark together with additional pions and/or quark-antiquark pairs form the unobserved state. In the first part of this work, the pionquark vertex is taken to be $g \gamma_{5} \tau_{i}$, where $\tau_{i}$ are the generators of the $\mathrm{SU}(2)$ flavor group. ${ }^{1}$ We assume the coupling

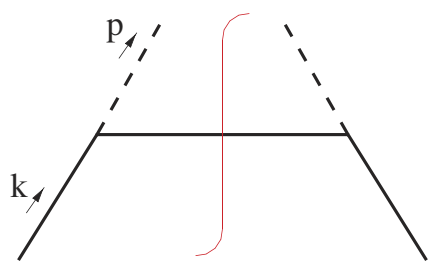

FIG. 1 (color online). Tree-level cut diagram describing the fragmentation of a quark into a pion. This diagram is common to all models, but the specific form of the pion-quark vertex can change. to be pointlike. This assumption is of course not appropriate at large transverse momenta of the pion. In fact, when integrating the fragmentation functions over $k_{T}$ divergences occur. Therefore, we impose a cutoff on the virtuality of the incoming quark, and study the dependence on the cutoff in some detail. A different approach would be to insert form factors. This could sensibly change the behavior of the fragmentation functions compared to our results.

Before entering the details of the calculation it is worthwhile to add some comments on the general philosophy underlying the model calculations. As a matter of principle, fragmentation functions cannot be computed by means of perturbative QCD. They either have to be fitted to data or computed in some effective approach to nonperturbative QCD. It is well known that in the low energy domain of QCD the Goldstone bosons, most notably the pions, play a crucial role. Therefore, in the model calculation of fragmentation functions they are considered as (effective) degrees of freedom, which at low scales appear in addition to the partonic degrees of freedom of QCD. This is, e.g., also the underlying picture of the chiral quark model of Manohar and Georgi [31] which we are going to use in the next section. In such an approach there is of course always a danger of double counting (for details on this issue we refer here also to Ref. [31]). On the other hand, one has to keep in mind that the gauge-link contribution to the fragmentation functions cannot directly be modeled by pion exchange, but rather the exchange of a spin-1 particle is required. Moreover, as we discuss in more detail below, pionic and gluonic contributions to the Collins function tend to have opposite signs. Therefore, in the case of our particular calculation of the Collins function we see no direct indication of double counting.

\section{A. Unpolarized fragmentation function}

We briefly reproduce the results already obtained in Ref. [19], but we present also a discussion of the parameter dependence of our results. Here and in the next sections, all results are for, e.g., the transition $u \rightarrow \pi^{0}$. An additional isospin factor of 2 has to be included for, e.g., the transition $u \rightarrow \pi^{+}$. The definition of the unpolarized fragmentation

\footnotetext{
${ }^{1}$ Note that in Ref. [19] the isospin structure was neglected, since it was not relevant to the purpose of that paper. This leads to different overall numerical factors in some of the final results.
} 
function is

$$
D_{1}\left(z, z^{2} \vec{k}_{T}^{2}\right)=\operatorname{Tr}\left[\Delta\left(z, \vec{k}_{T}\right) \gamma^{-}\right] .
$$

We compute the unpolarized fragmentation functions at tree level only, i.e. only using the diagram of Fig. 1. This is not entirely consistent with the fact that one-loop corrections need to be introduced in order to calculate the Collins function. We believe that the corrections to our final results will be small, though it would be appropriate to check in which kinematical region this statement holds. The result obtained from the calculation of the tree-level diagram is

$$
D_{1}\left(z, z^{2} \vec{k}_{T}^{2}\right)=\frac{1}{z} \frac{g^{2}}{16 \pi^{3}} \frac{\vec{k}_{T}^{2}+m^{2}}{\left(\vec{k}_{T}^{2}+m^{2}+\frac{1-z}{z^{2}} m_{\pi}^{2}\right)^{2}} .
$$

The integrated unpolarized fragmentation function $D_{1}(z)$ is defined as

$$
D_{1}(z)=\pi \int_{0}^{\vec{K}_{T \max }^{2}} d \vec{K}_{T}^{2} D_{1}\left(z, \vec{K}_{T}^{2}\right),
$$

where $\vec{K}_{T}=-z \vec{k}_{T}$ denotes the transverse momentum of the outgoing hadron with respect to the quark direction. The upper limit on the $\vec{K}_{T}^{2}$ integration is set by the cutoff on the fragmenting quark virtuality, $\mu^{2}$, and corresponds to

$$
\vec{K}_{T \max }^{2}=z(1-z) \mu^{2}-z m^{2}-(1-z) m_{\pi}^{2} .
$$

The analytic result for the integrated fragmentation function is

$$
\begin{aligned}
D_{1}(z)= & \frac{g^{2}}{16 \pi^{2}}\left[z \ln \left(\frac{(1-z)\left(\mu^{2}-m^{2}\right)}{z\left(m^{2}+m_{\pi}^{2} \frac{1-z}{z^{2}}\right)}\right)\right. \\
& -\left(z(1-z) \mu^{2}-z m^{2}-(1-z) m_{\pi}^{2}\right) \\
& \left.\times \frac{m_{\pi}^{2}}{z^{2}\left(\mu^{2}-m^{2}\right)\left(m^{2}+m_{\pi}^{2} \frac{1-z}{z^{2}}\right)}\right] .
\end{aligned}
$$

In Fig. 2 we show the result of the model calculation of the function $D_{1}^{u \rightarrow \pi^{+}}$for a choice of the coupling constant

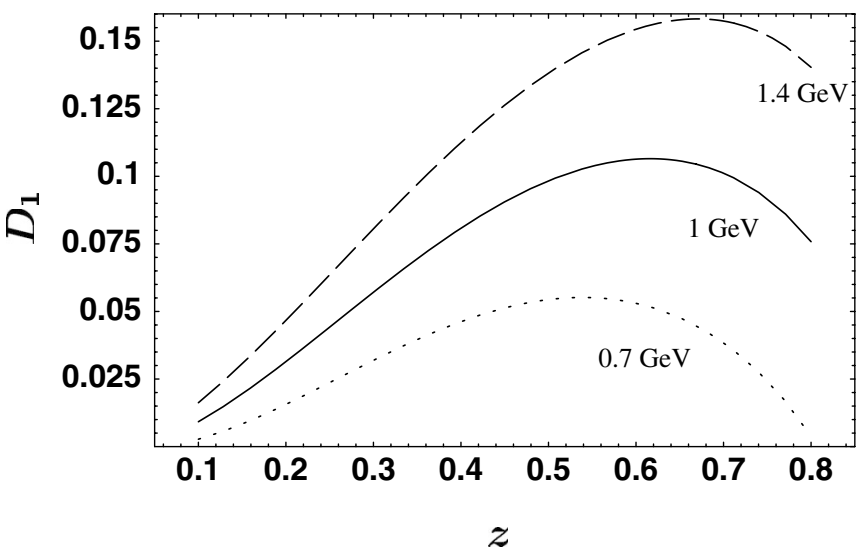

$g=3$ and for different values of the parameters $\mu$ and $m$. Our value for $g$ is about $1 / 3$ of the pseudoscalar pionnucleon coupling, which can be considered as a reasonable choice. Of course this value is not extremely well determined. Nevertheless, most of the pertinent results like the $z$-shape of fragmentation functions, and the relative magnitude of various contributions to the Collins function are not very sensitive to the precise value of $g$. Keeping in mind this large value of $g$ it is quite possible that higher order corrections to the calculation of $D_{1}$ can be significant. However, without performing an explicit calculation of such corrections one cannot make a definite statement about their numerical importance.

From Fig. 2 we deduce that, apart from the trivial dependence on the coupling strength, an increase of the cutoff or a decrease of the quark mass makes the fragmentation function bigger, without sensibly changing the $z$ dependence. The shape of the unpolarized fragmentation function is very far from standard parametrizations extracted from phenomenology (see, e.g. Ref. [32]), even from a qualitative point of view. As mentioned before, different behaviors can be obtained by modifying the model through the insertion of form factors, as can be seen comparing our results with those of Ref. [21].

\section{B. Collins function from pion loops}

We use the following definition of the Collins function [2], in agreement with the "Trento conventions" [33],

$$
\frac{\epsilon_{T}^{i j} k_{T j}}{m_{\pi}} H_{1}^{\perp}\left(z, z^{2} \vec{k}_{T}^{2}\right)=\operatorname{Tr}\left[\Delta\left(z, \vec{k}_{T}\right) i \sigma^{i-} \gamma_{5}\right] .
$$

The Collins function receives contributions only from the interference between two amplitudes with different imaginary parts. In our case, the tree-level amplitude is real and the necessary imaginary parts are generated by the inclusion of one-loop corrections. Such corrections contain imaginary parts if and only if it is kinematically possible that the particles in the loop go on shell. In this section, we

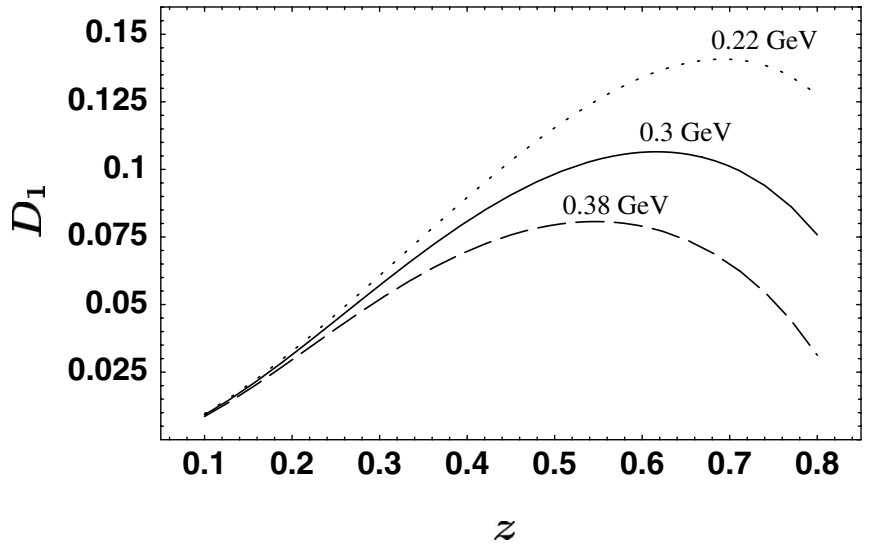

FIG. 2. Unpolarized fragmentation function $D_{1}^{u \rightarrow \pi^{+}}$in a fragmentation model with pseudoscalar pion-quark coupling. Left panel: dependence on the parameter $\mu$ (for $m=0.3 \mathrm{GeV}$ ). Right panel: dependence on the parameter $m$ (for $\mu=1 \mathrm{GeV}$ ). 
will make use of pion loops. Keeping in mind that the initial quark is virtual and timelike, the only two diagrams contributing to the Collins function at one loop are the ones shown in Fig. 3, plus their Hermitian conjugates. We refer to these two diagrams as self-energy (a) and vertex (b) corrections.

The explicit calculation of the one-loop diagrams leads to the following result for the Collins function:

$$
\begin{aligned}
H_{1}^{\perp}\left(z, z^{2} \vec{k}_{T}^{2}\right)= & -\frac{g^{2}}{8 \pi^{3}} \frac{m_{\pi}}{1-z} \frac{m}{k^{2}-m^{2}} \\
& \times\left.\left(\operatorname{Im} \sigma_{P S}^{\pi}+\operatorname{Im} \gamma_{1, P S}^{\pi}\right)\right|_{k^{2}=\vec{k}_{T \frac{z}{1-z}}^{2}+\frac{m^{2}}{1-z}+\frac{m_{\pi}^{2}}{z}}
\end{aligned}
$$

where we distinguished the contributions from diagram (a) and (b), being respectively

$$
\begin{aligned}
\operatorname{Im} \sigma_{P S}^{\pi}= & \frac{3 g^{2}}{16 \pi^{2}} \frac{1}{k^{2}-m^{2}}\left(1-\frac{m^{2}-m_{\pi}^{2}}{k^{2}}\right) I_{1, \pi}, \\
\operatorname{Im} \gamma_{1, P S}^{\pi}= & \frac{g^{2}}{8 \pi^{2}} \frac{k^{2}-m^{2}+m_{\pi}^{2}}{\lambda_{\pi}} \\
& \times\left(I_{1, \pi}+\left(k^{2}-m^{2}-2 m_{\pi}^{2}\right) I_{2, \pi}\right) .
\end{aligned}
$$

In the above formulae we have used the integrals

$$
\begin{gathered}
I_{1, \pi}=\int d^{4} l \delta\left(l^{2}-m_{\pi}^{2}\right) \delta\left((k-l)^{2}-m^{2}\right) \\
=\frac{\pi}{2 k^{2}} \sqrt{\lambda_{\pi}} \theta\left(k^{2}-\left(m+m_{\pi}\right)^{2}\right), \\
I_{2, \pi}=\int d^{4} l \frac{\delta\left(l^{2}-m_{\pi}^{2}\right) \delta\left((k-l)^{2}-m^{2}\right)}{(k-p-l)^{2}-m^{2}} \\
=-\frac{\pi}{2 \sqrt{\lambda_{\pi}}} \ln \left(1+\frac{\lambda_{\pi}}{k^{2} m^{2}-\left(m^{2}-m_{\pi}^{2}\right)^{2}}\right) \\
\quad \times \theta\left(k^{2}-\left(m+m_{\pi}\right)^{2}\right),
\end{gathered}
$$

and the definition $\lambda_{\pi}=\lambda\left(k^{2}, m^{2}, m_{\pi}^{2}\right)=\left[k^{2}-(m+\right.$ $\left.\left.m_{\pi}\right)^{2}\right]\left[k^{2}-\left(m-m_{\pi}\right)^{2}\right]$.

Comparing these results with the original publication [19], we notice that there is an overall sign error in the final result for the Collins function. Furthermore, due to the introduction of the isospin structure, which was neglected in the original work, the contribution of the self-energy correction, Eq. (9), has an extra factor 3, while the con-

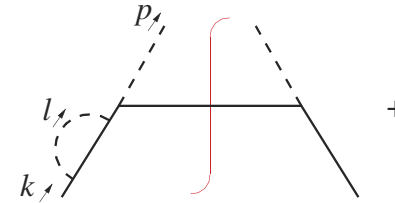

(a)

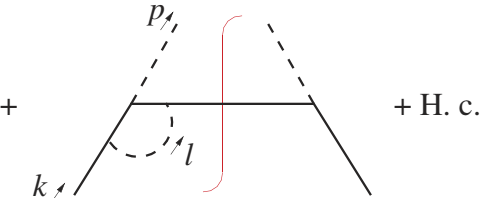

(b)
FIG. 3 (color online). Single pion-loop corrections to the fragmentation of a quark into a pion.

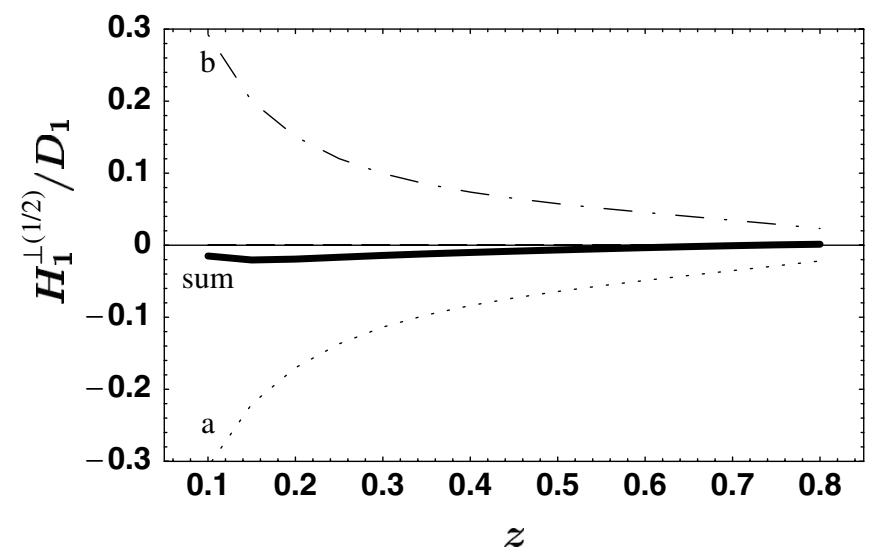

FIG. 4. Contributions to $H_{1}^{\perp(1 / 2)} / D_{1}$ from the diagrams of Fig. 3 and their sum.

tribution of the vertex correction, Eq. (10), has a different sign.

In Fig. 4 we present numerical estimates of the following quantity

$$
\frac{H_{1}^{\perp(1 / 2)}(z)}{D_{1}(z)} \equiv \frac{\pi}{D_{1}(z)} \int d \vec{K}_{T}^{2} \frac{\left|\vec{K}_{T}\right|}{2 z m_{\pi}} H_{1}^{\perp}\left(z, \vec{K}_{T}^{2}\right),
$$

separately for each diagram in Fig. 3. The most prominent feature to be noticed is that the contributions of diagrams (a) and (b) have similar size but opposite signs, causing a strong cancellation and giving rise to a very small Collins function. We checked that this behavior is persistent even when changing the parameters $m$ and $\mu$.

\section{Collins function from gluon loops}

Instead of using pion rescattering as a source of imaginary parts, it is possible to consider gluon single-loop corrections. In fact, gluon exchange is essential to ensure color gauge invariance of the fragmentation functions. The diagrams involved in the calculation of the Collins function at the one-loop level are drawn in Fig. 5. The self-energy (a) and vertex (b) corrections are analogous to the previous case. The last two diagrams represent the interaction with the quark before being struck by the photon in semiinclusive DIS (SIDIS), or with the outgoing antiquark in the case of $e^{+} e^{-}$annihilation. We can call them the photon-vertex (c) and box diagram (d) corrections. At leading order in $1 / Q, Q$ being the virtuality of the photon, the eikonal approximation can be applied, the quark (antiquark) can be replaced by an eikonal line and the gluon interaction can be factorized and included in the definition of the correlation function, giving rise precisely to the gauge links appearing in Eq. (1). When performing QCD factorization for a diagram representing the full semiinclusive DIS or $e^{+} e^{-}$annihilation process, the hard photon-quark vertex is separated from the soft physics encoded in the fragmentation correlator shown in Fig. 5. 


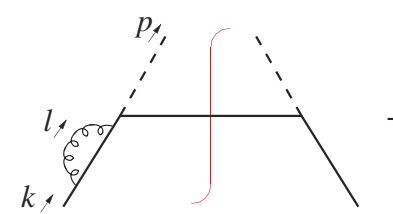

(a)

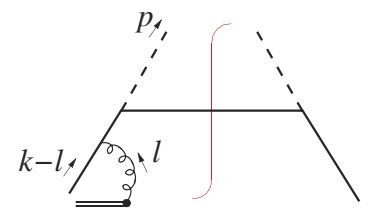

(c)

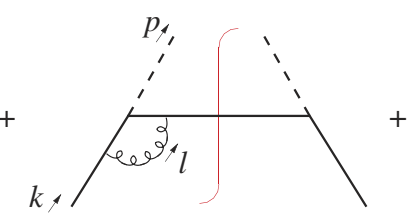

(b)

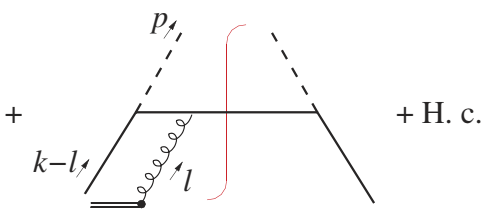

(d)
FIG. 5 (color online). Single gluon-loop corrections to the fragmentation of a quark into a pion. The double line in (c) and (d) represents the eikonalized quark propagator.
The eikonal approximation means that one keeps the leading term, in an expansion in powers of $1 / Q$, of the quark (antiquark) propagator in the case where the gluon is collinear to the pion (or remnants) in the final state. If the gluon has a different momentum configuration one gets, at least for the box diagram, a power suppressed contribution. The eikonalized propagators give rise to the factors $1 /\left(-l^{-} \pm i \varepsilon\right)$ in Eqs. (22) and (23). The sign of the $i \varepsilon$ term depends on whether we deal with SIDIS or $e^{+} e^{-}$ annihilation, respectively, but it does not influence the result for the Collins function. The procedure of the eikonal approximation and factorization has been discussed in detail for distribution functions [26,29,30,34], and for fragmentation functions [27].

As an illustration of the use of the eikonal approximation, we give the explicit formula for the contribution of diagram (d) of Fig. 5 to the correlation function

$$
\begin{aligned}
\Delta_{(d)}(k, p)= & \frac{1}{(2 \pi)^{4}} \frac{i(k+m)}{k^{2}-m^{2}} g \gamma_{5}(k-\not k+m) 2 \pi \delta\left((k-p)^{2}-m^{2}\right) \\
& \times \int \frac{d^{4} l}{(2 \pi)^{4}} \frac{i g_{S} \gamma_{\mu} t^{a} i(k-\not k-\not l+m) g \gamma_{5} i(k-\not l+m) i\left(-i g^{\mu-}\right)\left(i g_{S} t^{a}\right)}{\left((k-p-l)^{2}-m^{2}+i \varepsilon\right)\left((k-l)^{2}-m^{2}+i \varepsilon\right)\left(-l^{-} \pm i \varepsilon\right)\left(l^{2}-m_{g}^{2}+i \varepsilon\right)},
\end{aligned}
$$

where $t^{a}$ are the generators of the $\mathrm{SU}(3)$ color group.

The Collins function obtained by the calculations of the diagrams in Fig. 5 can be written as

$$
\begin{aligned}
H_{1}^{\perp}\left(z, z^{2} \vec{k}_{T}^{2}\right)= & -\frac{g^{2}}{8 \pi^{3}} \frac{m_{\pi}}{1-z} \frac{m}{k^{2}-m^{2}}\left(\operatorname{Im} \sigma_{P S}^{g}+\operatorname{Im} \gamma_{1, P S}^{g}\right. \\
& \left.+\operatorname{Im} \phi_{P S}+\operatorname{Im} \xi_{P S}\right)\left.\right|_{k^{2}=\vec{k}_{T}^{2} \frac{z}{1-z}+\frac{m^{2}}{1-z}+\frac{m_{\pi}^{2}}{z}}
\end{aligned}
$$

where we distinguished the contributions from diagram (a), (b), (c), and (d), being respectively

$$
\begin{gathered}
\operatorname{Im} \sigma_{P S}^{g}=\frac{\alpha_{s}}{2 \pi} C_{F} \frac{1}{k^{2}-m^{2}}\left(3-\frac{m^{2}}{k^{2}}\right) I_{1, g}, \\
\operatorname{Im} \gamma_{1, P S}^{g}=\frac{\alpha_{s}}{2 \pi} 2 C_{F} I_{2, g}, \\
\operatorname{Im} \phi_{P S}=0,
\end{gathered}
$$

$$
\begin{aligned}
\operatorname{Im} \xi_{P S}= & -\frac{\alpha_{s}}{2 \pi} C_{F} \frac{1}{z \vec{k}_{T}^{2}}\left(z k^{-}\left(\tilde{I}_{3, g}+(1-z)\left(k^{2}-m^{2}\right) \tilde{I}_{4, g}\right)\right. \\
& \left.-\left(z\left(k^{2}-m^{2}+m_{\pi}^{2}\right)-2 m_{\pi}^{2}\right) I_{2, g}\right)
\end{aligned}
$$

These results are valid only for the case $m_{g}=0$.

Equation (18) shows that no contribution to the Collins function arises from the photon-vertex correction of diagram (c). Even if it is kinematically possible to have an imaginary part in this diagram, it cannot contribute to the nontrivial Dirac structure connected to the Collins function.

In the above formulae we have used the integrals

$$
\begin{aligned}
I_{1, g} & =\int d^{4} l \delta\left(l^{2}\right) \delta\left((k-l)^{2}-m^{2}\right) \\
& =\frac{\pi}{2 k^{2}}\left(k^{2}-m^{2}\right) \theta\left(k^{2}-m^{2}\right),
\end{aligned}
$$

$$
\begin{gathered}
I_{2, g}=\int d^{4} l \frac{\delta\left(l^{2}\right) \delta\left((k-l)^{2}-m^{2}\right)}{(k-p-l)^{2}-m^{2}} \\
=-\frac{\pi}{2 \sqrt{\lambda_{\pi}}} \ln \left(1+\frac{2 \sqrt{\lambda_{\pi}}}{k^{2}+m^{2}-m_{\pi}^{2}-\sqrt{\lambda_{\pi}}}\right) \\
\times \theta\left(k^{2}-m^{2}\right), \\
\tilde{I}_{3, g}=\int d^{4} l \frac{\delta\left(l^{2}\right) \delta\left((k-l)^{2}-m^{2}\right)}{-n \cdot l \pm i \varepsilon}, \\
\tilde{I}_{4, g}=\int d^{4} l \frac{\delta\left(l^{2}\right) \delta\left((k-l)^{2}-m^{2}\right)}{\left[(k-p-l)^{2}-m^{2}\right](-n \cdot l \pm i \varepsilon)} .
\end{gathered}
$$

The lightlike vector $n$ in the integrals $\tilde{I}_{3, g}$ and $\tilde{I}_{4, g}$ is defined via $n \cdot a=a^{-}$for an arbitrary 4-vector $a^{\mu}$. For our purpose, we need only the following linear combination of the last two integrals

$$
\tilde{I}_{3, g}+(1-z)\left(k^{2}-m^{2}\right) \tilde{I}_{4, g}=\frac{\pi}{k^{-}} \ln \frac{\sqrt{k^{2}}(1-z)}{m} .
$$


From Eqs. (22) and (23) it is evident that the contribution to the Collins function coming from diagram (d) in Fig. 5 does not come from the pole of the eikonal propagator, but rather from the cut crossing the gluon and the incoming quark. In fact, a contribution from the eikonal propagator would imply a violation of universality of the fragmentation functions [28]. This is in our opinion a model-independent statement, merely due to kinematical conditions, in agreement with the general statements of Ref. [27].

At this point, we would like to comment on the model calculation presented in Ref. [21]. The results about diagram (d) obtained by the authors in that paper are in disagreement with ours, even when taking the pointlike limit of the Gaussian form factor. We believe that this is due to a mistake in that paper. The Collins function obtained there, Eq. (11), is erroneously coming from the imaginary part of the eikonal propagator. This is evident also by comparison with the results obtained for the distribution function $h_{1}^{\perp}$ in Ref. [35], Eq. (16). Taking the pointlike form-factor limit of the Collins function of Ref. [21], it turns out to be related in a simple way to the distribution function $h_{1}^{\perp}$ in Ref. [35]. Such a relation should not hold, even if only diagram (d) is taken into account, since, as already mentioned before, the two functions receive contributions from different cuts: the cut crossing the eikonal line and the outgoing antiquark in the distribution function, and the cut crossing the gluon and incoming quark in the fragmentation function.

In Fig. 6, we present numerical estimates for the quantity $H_{1}^{\perp(1 / 2)} / D_{1}$, separately for each of the diagrams of Fig. 5. It is interesting to note that also in this case there is a strong cancellation between the contribution of diagrams (a) and (b), to a large extent independent of the parameter choice. At large values of $z$, the dominant part comes therefore from diagram (d), the gauge-link box diagram. This observation may give support to the idea of calculating the Collins function taking into account only this diagram, as done in Ref. [21].

\section{PSEUDOVECTOR PION-QUARK COUPLING}

The tree-level diagram to be used for the calculation of the unpolarized fragmentation function is the same as before (Fig. 1). However, now the pion-quark vertex is taken to be $g_{A} /\left(2 F_{\pi}\right) \gamma_{5} \not p$, where $p$ is the momentum of the outgoing pion, as drawn in the picture. When working with on-shell particles, the pseudoscalar and pseudovector couplings are known to be equivalent. Here, however, we are dealing with an off-shell fragmenting quark and the two versions of the coupling lead to completely different results. Chiral invariance of the model is obtained by including also a $\pi \pi q q$ contact interaction [20,31]. As we discuss below the contact term dominates the numerics of the Collins function to a large extent. As before, we use a pointlike pion-quark coupling, without form factors, but

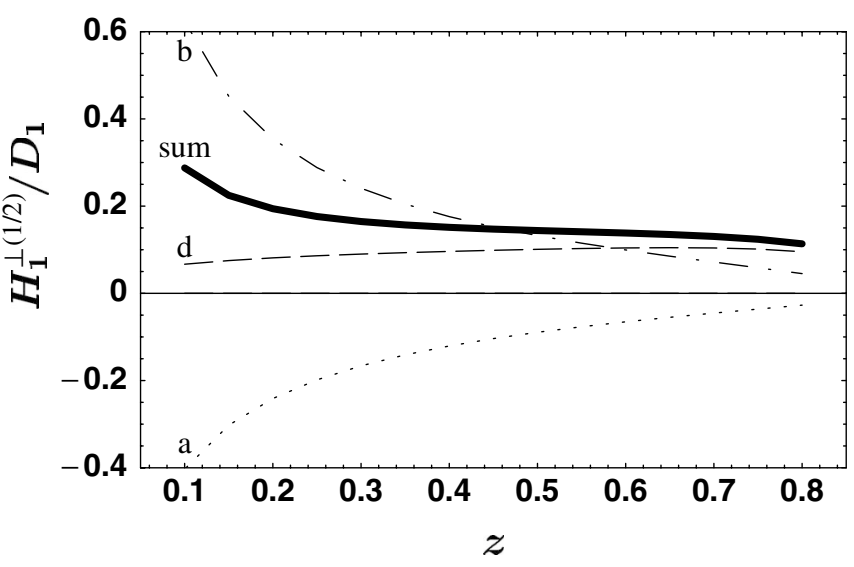

FIG. 6. Contributions to $H_{1}^{\perp(1 / 2)} / D_{1}$ from the diagrams of Fig. 5 and their sum.

we impose a cutoff in the virtuality of the incoming quark to avoid divergences. So far, no inclusion of form factors was ever attempted on this type of model.

\section{A. Unpolarized fragmentation function}

In this section, we compute the unpolarized fragmentation function $D_{1}$ at tree level, as done in Ref. [20], adding a few comments on the parameter dependence of the outcome. Again, all results are for, e.g., the transition $u \rightarrow \pi^{0}$ and an additional isospin factor of 2 has to be included for, e.g., the transition $u \rightarrow \pi^{+}$. The calculation of the treelevel cut diagram of Fig. 1 yields the result

$$
\begin{aligned}
D_{1}\left(z, z^{2} \vec{k}_{T}^{2}\right)= & \frac{1}{z} \frac{g_{A}^{2}}{4 F_{\pi}^{2}} \frac{1}{16 \pi^{3}}\left(1-4 \frac{1-z}{z^{2}}\right. \\
& \left.\times \frac{m^{2} m_{\pi}^{2}}{\left[\vec{k}_{T}^{2}+m^{2}+\frac{1-z}{z^{2}} m_{\pi}^{2}\right]^{2}}\right) .
\end{aligned}
$$

We integrate over $z \vec{k}_{T}$ in the same manner as done in Sec. II A, and obtain the integrated fragmentation function $D_{1}(z)$

$$
\begin{aligned}
D_{1}(z)= & \frac{g_{A}^{2}}{64 F_{\pi}^{2} \pi^{2}}\left(z(1-z) \mu^{2}-z m^{2}-(1-z) m_{\pi}^{2}\right) \\
& \times\left(\frac{1}{z}-\frac{4 m^{2} m_{\pi}^{2}}{z^{2}\left(\mu^{2}-m^{2}\right)\left(m^{2}+m_{\pi}^{2} \frac{1-z}{z^{2}}\right)}\right) .
\end{aligned}
$$

In Fig. 7 we show the result of the model calculation of the function $D_{1}^{u \rightarrow \pi^{+}}$for $g_{A}=1$ and $F_{\pi}=93 \mathrm{MeV}$. We note that an increase of the value of the cutoff parameter $\mu$ makes the function bigger, without sensibly changing the $z$ dependence. The dependence on the constituent quark mass $m$ is weak. These features were already commented on in Ref. [20], where the values of $\mu=1 \mathrm{GeV}$ and $m=$ $0.3 \mathrm{GeV}$ were selected as best choices. This decision was justified by comparison with a standard parametrization of the function $D_{1}^{u \rightarrow \pi^{+}}-D_{1}^{\bar{u} \rightarrow \pi^{+}}$and the experimental data 

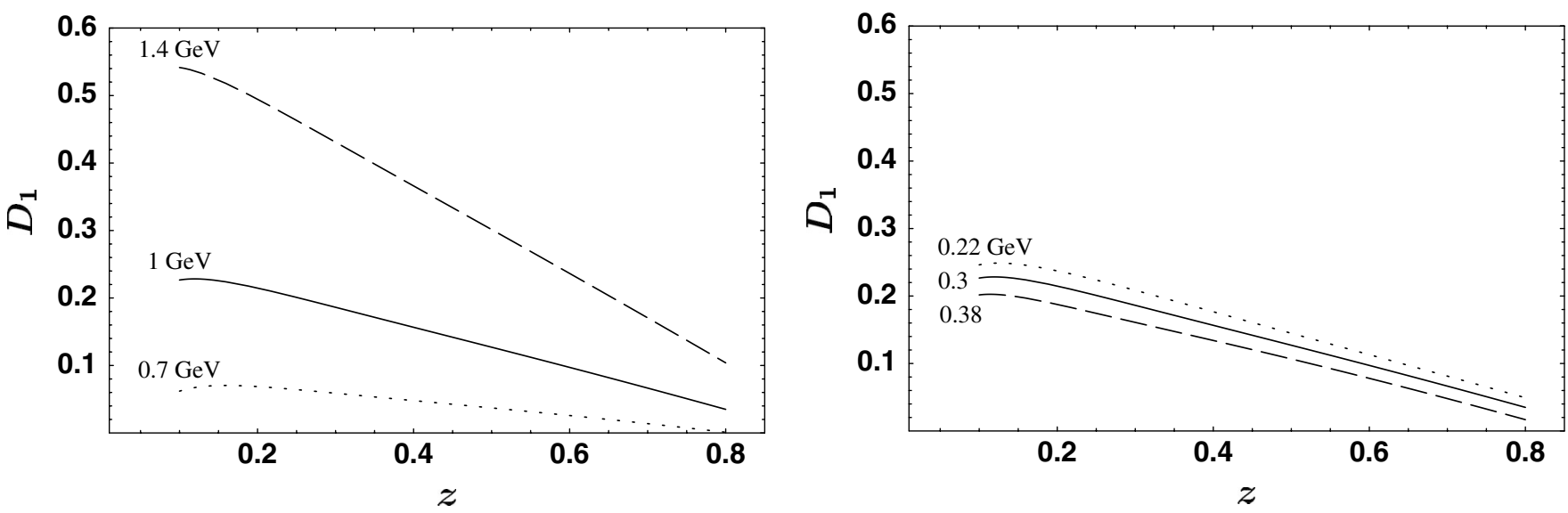

FIG. 7. Unpolarized fragmentation function $D_{1}^{u \rightarrow \pi^{+}}$in a fragmentation model with pseudovector pion-quark coupling. Left panel: dependence on the parameter $\mu$ (for $m=0.3 \mathrm{GeV}$ ). Right panel: dependence on the parameter $m$ (for $\mu=1 \mathrm{GeV}$ ).

on the average transverse momentum of hadrons in fragmentation processes.

\section{B. Collins function from pion loops}

After the introduction of single pion-loop corrections, all the diagrams contributing to the Collins function are depicted in Fig. 8. As mentioned before, apart from the self-energy and vertex corrections, chiral invariance requires the contact-interaction term, diagram (c), which turns out to be dominant on the others.

The resulting Collins function can be written in a compact form as

$$
\begin{aligned}
H_{1}^{\perp}\left(z, z^{2} \vec{k}_{T}^{2}\right)= & \frac{g_{A}^{2}}{32 \pi^{3} F_{\pi}^{2}} \frac{m_{\pi}}{1-z} \frac{m}{k^{2}-m^{2}}\left(\operatorname{Im} \sigma_{P V}^{\pi}\right. \\
& \left.+\operatorname{Im} \gamma_{1, P V}^{\pi}+\operatorname{Im} \gamma_{2, P V}^{\pi}\right)\left.\right|_{k^{2}=\vec{k}_{T 1-z}^{2} \frac{z}{1-z}+\frac{m^{2}}{1-z}+\frac{m_{\pi}^{2}}{z}}
\end{aligned}
$$

The three terms correspond, respectively, to the contributions of diagrams (a), (b), (c) in Fig. 8, and read

$$
\begin{aligned}
\operatorname{Im} \sigma_{P V}^{\pi}= & \frac{3 g_{A}^{2}}{32 \pi^{2} F_{\pi}^{2}}\left(2 m_{\pi}^{2}-\frac{1}{2}\left(k^{2}-m^{2}\right)\right. \\
& \left.\times\left(1-\frac{m^{2}-m_{\pi}^{2}}{k^{2}}\right)\right) I_{1, \pi}
\end{aligned}
$$

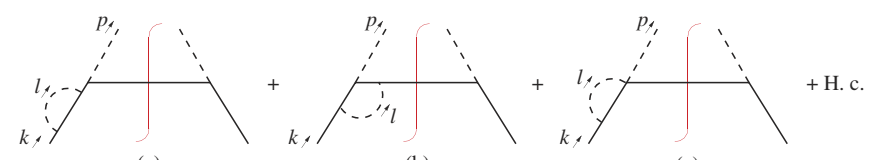

(a)

(b)

FIG. 8 (color online). Single pion-loop corrections to the fragmentation of a quark into a pion.

$$
\begin{aligned}
\operatorname{Im} \gamma_{1, P V}^{\pi}= & \frac{g_{A}^{2}}{32 \pi^{2} F_{\pi}^{2}}\left(k^{2}-m^{2}\right)\left(\frac{1}{2 k^{2}}\left(3 k^{2}+m^{2}-m_{\pi}^{2}\right) I_{1, \pi}\right. \\
& +4 m^{2} \frac{k^{2}-m^{2}+m_{\pi}^{2}}{\lambda_{\pi}}\left(I_{1, \pi}+\left(k^{2}-m^{2}\right.\right. \\
& \left.\left.\left.-2 m_{\pi}^{2}\right) I_{2, \pi}\right)\right)
\end{aligned}
$$

$$
\operatorname{Im} \gamma_{2, P V}^{\pi}=-\frac{2}{32 \pi^{2} F_{\pi}^{2}}\left(k^{2}-m^{2}\right)\left(1-\frac{m^{2}-m_{\pi}^{2}}{k^{2}}\right) I_{1, \pi} .
$$

We point out that in the original publication [20] a sign error was made. The sign of all results for the Collins function should be reversed.

In Fig. 9 we present numerical estimates of the ratio $H_{1}^{\perp(1 / 2)} / D_{1}$, separately for each diagram in Fig. 8 . As in the previous cases, also in the present one the contribution from diagrams (a) and (b) (i.e. self-energy and vertex corrections) roughly cancel each other. The dominant contribution to the Collins function comes therefore from diagram (c), i.e. the contact-interaction diagram. As already mentioned before, the result of the sum of diagrams correspond to that obtained in Ref. [20] (Fig. 8) except for the overall sign.

\section{Collins function from gluon loops}

As discussed in Sec. II C, we can use gluon-loop corrections to generate imaginary parts in the diagrams. The Collins function becomes

$$
\begin{aligned}
H_{1}^{\perp}\left(z, z^{2} \vec{k}_{T}^{2}\right)= & \frac{g_{A}^{2}}{32 \pi^{3} F_{\pi}^{2}} \frac{m_{\pi}}{1-z} \frac{m}{k^{2}-m^{2}} \\
& \times\left(\operatorname{Im} \sigma_{P V}^{g}+\operatorname{Im} \gamma_{1, P V}^{g}\right. \\
& \left.+\operatorname{Im} \phi_{P V}+\operatorname{Im} \xi_{P V}\right)\left.\right|_{k^{2}=\vec{k}_{T}^{2} \frac{z}{1-z}+\frac{m^{2}}{1-z}+\frac{m_{\pi}^{2}}{z}}
\end{aligned}
$$




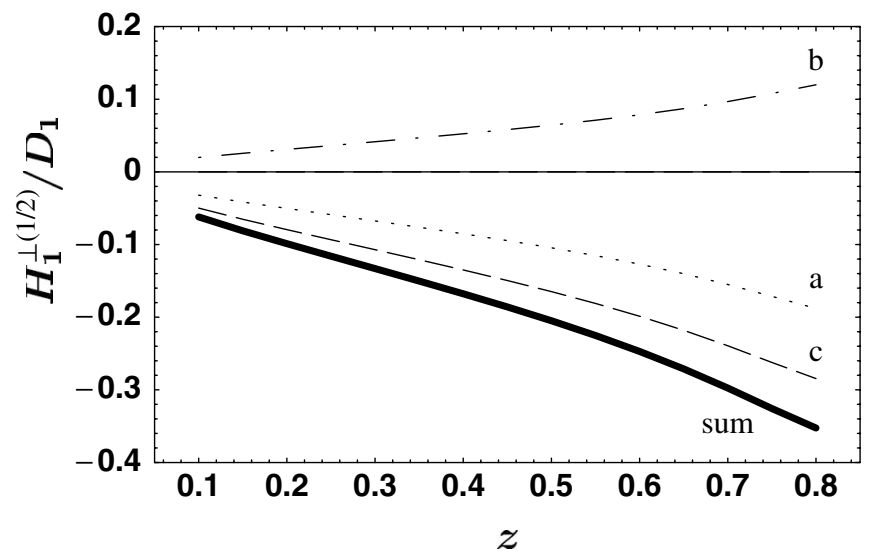

FIG. 9. Contributions to $H_{1}^{\perp(1 / 2)} / D_{1}$ from the diagrams of Fig. 8 and their sum.

where each term represents the contribution of one of the diagrams in Fig. 5, and reads

$$
\begin{gathered}
\operatorname{Im} \sigma_{P V}^{g}=\frac{\alpha_{s}}{2 \pi} C_{F}\left(3-\frac{m^{2}}{k^{2}}\right) I_{1, g}, \\
\operatorname{Im} \gamma_{1, P V}^{g}=-\frac{\alpha_{s}}{2 \pi} C_{F}\left(\left(1+\frac{m^{2}}{k^{2}}\right) I_{1, g}+4 m_{\pi}^{2} I_{2, g}\right),
\end{gathered}
$$$$
\operatorname{Im} \phi_{P V}=0,
$$

$$
\begin{aligned}
\operatorname{Im} \xi_{P V}= & \frac{\alpha_{s}}{\pi} C_{F}\left(2 I_{1, g}+2 k^{-}\left(\tilde{I}_{3, g}+(1-z)\left(k^{2}-m^{2}\right) \tilde{I}_{4, g}\right)\right. \\
& +\frac{2 z m^{2}-(1-z)\left(k^{2}-m^{2}\right)}{z^{2} \vec{k}_{T}^{2}}\left(z k ^ { - } \left(\tilde{I}_{3, g}+(1-z)\right.\right. \\
& \left.\times\left(k^{2}-m^{2}\right) \tilde{I}_{4, g}\right)-\left(z\left(k^{2}-m^{2}+m_{\pi}^{2}\right)\right. \\
& \left.\left.\left.-2 m_{\pi}^{2}\right) I_{2, g}\right)\right) .
\end{aligned}
$$

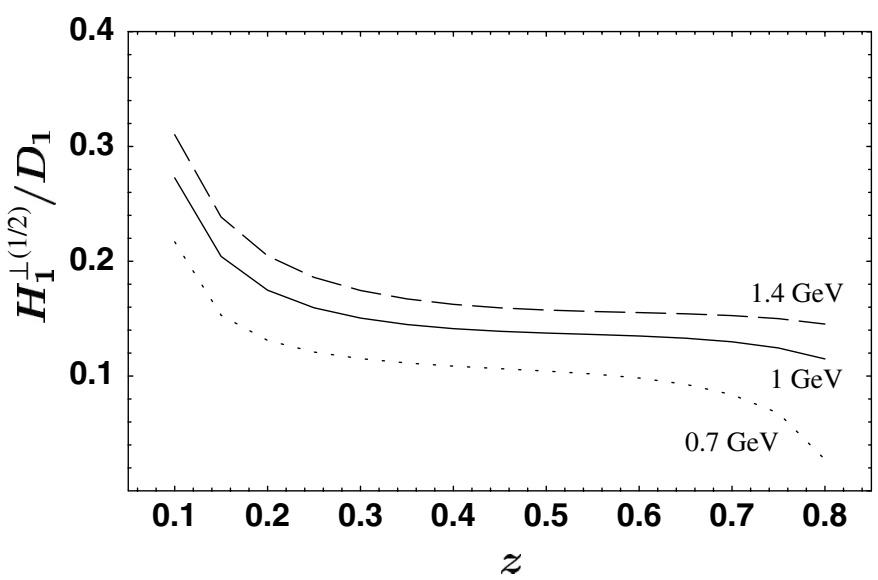

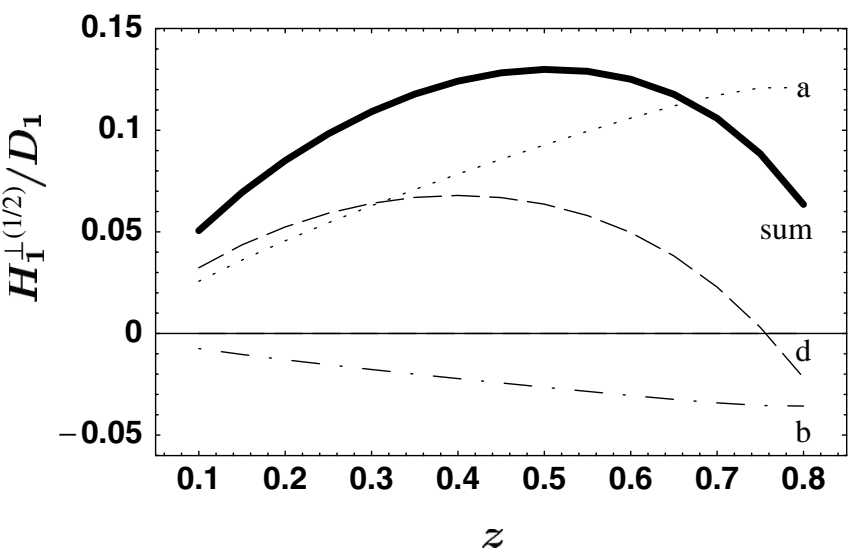

FIG. 10. Contributions to $H_{1}^{\perp(1 / 2)} / D_{1}$ from the diagrams of Fig. 5 and their sum.

The above results are valid only for the case $m_{g}=0$. Note the presence of the same linear combination of $\tilde{I}_{3, g}$ and $\tilde{I}_{4, g}$ defined in Eq. (24). There is again no contribution from the photon-vertex correction of diagram (c). Finally, there is no contribution to the Collins function arising from the pole in the eikonal propagator. We point out that in the original publication [22] a sign error was made and the sign of the results for the Collins function should be reversed.

Figure 10 shows the numerical estimates for the quantity $H_{1}^{\perp(1 / 2)} / D_{1}$, separately for each of the diagrams of Fig. 5 . This case is different from the previous ones, as no strong cancellation between the different diagrams takes place. The contribution from the vertex correction is opposite but much smaller than that from the self-energy correction, a feature persisting also for different values of the parameters. Hence, all contributions turn out to be important. The result of the sum of diagrams correspond to that obtained in Ref. [22] (Fig. 4) except for the overall sign. This completes our review of the different models to calculate the

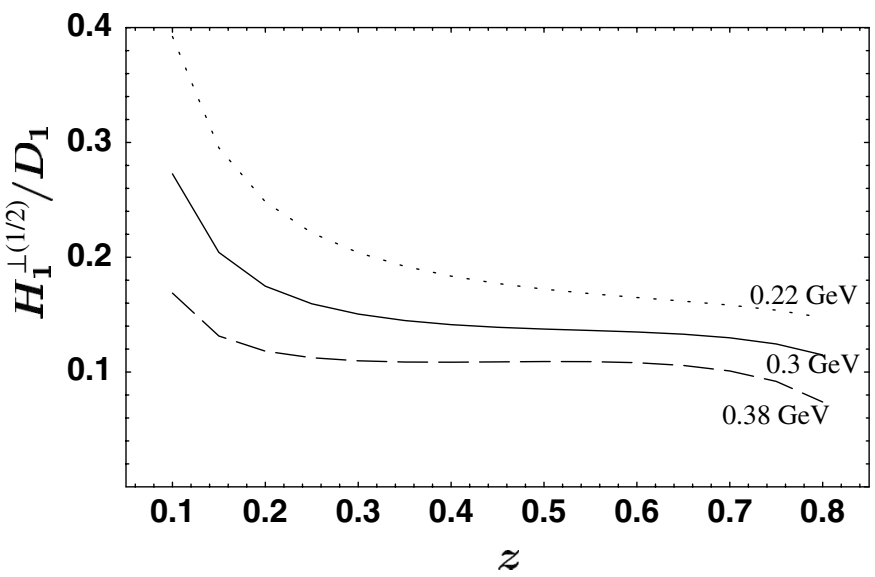

FIG. 11. Estimate of $H_{1}^{\perp(1 / 2)} / D_{1}$ in a model with pseudoscalar pion-quark interaction, including pion and gluon single-loop corrections. Left panel: dependence on the parameter $\mu$ (for $m=0.3 \mathrm{GeV}$ ). Right panel: dependence on the parameter $m$ (for $\mu=$ $1 \mathrm{GeV})$. 

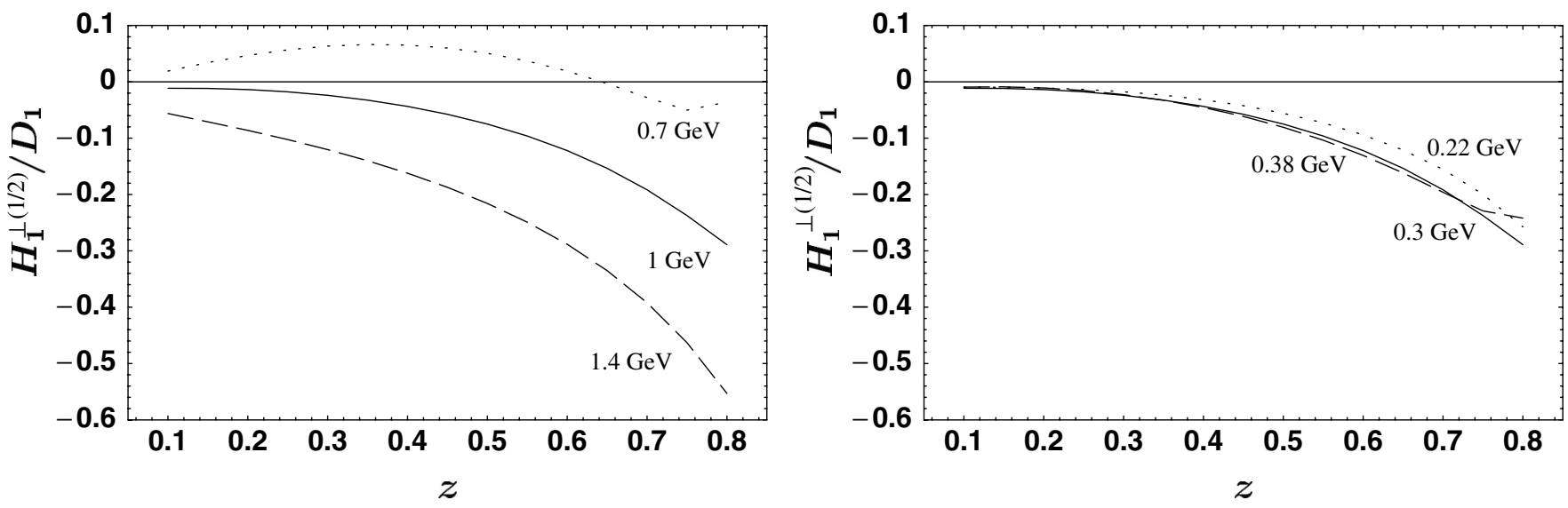

FIG. 12. Estimate of $H_{1}^{\perp(1 / 2)} / D_{1}$ in a model with pseudovector pion-quark interaction, including pion and gluon single-loop corrections. Left panel: dependence on the parameter $\mu$ (for $m=0.3 \mathrm{GeV}$ ). Right panel: dependence on the parameter $m$ (for $\mu=$ $1 \mathrm{GeV})$.

Collins function and the analysis of the contributions from each separate diagram. We turn now to some numerical results.

\section{NUMERICAL RESULTS AND ASYMMETRIES}

In this section, we present numerical results for the quantity $H_{1}^{\perp(1 / 2)} / D_{1}$, for different values of the relevant parameters $m$ and $\mu$, separately for the pseudoscalar and pseudovector pion-quark coupling. All possible contributions are summed, coming from pion loops as well as from gluon loops.

Figure 11 shows the result for the pseudoscalar coupling model of Sec. II. The left panel shows the dependence on the cutoff parameter $\mu$ and the right panel the dependence on the constituent quark mass $m$. In this model the dominant part of the Collins function arises from gluon interactions, in particular, from the gauge-link box diagram, as can be seen by comparing the solid lines in Fig. 11 with that in Fig. 6. In general, this model gives rise to a ratio $H_{1}^{\perp(1 / 2)} / D_{1}$ of about $10 \%-20 \%$, with a flat dependence on the variable $z$ in the range $0.2 \leq z \leq 0.8$.

Figure 12 shows the result for the pseudovector coupling model of Sec. III. As before, the dependence on the cutoff parameter $\mu$ and on the constituent quark mass $m$ is shown in the left and right panel, respectively. In this case, for higher values of the parameter $\mu$ the contact-interaction contribution, diagram (c) of Fig. 8, is the dominant one, making the ratio $H_{1}^{\perp(1 / 2)} / D_{1}$ negative and as big as $30 \%-$ $50 \%$. However, as the value of $\mu$ is reduced, the size of the contact-interaction contribution decreases, while the size of the contributions from the gluon loops increase. The value of the ratio $H_{1}^{\perp(1 / 2)} / D_{1}$ can in this case become positive.

In order to allow a more direct comparison with experimental results, we estimated the Collins transverse singlespin asymmetry for $\pi^{+}$production in semi-inclusive DIS.
We use the definitions of the asymmetries and of the azimuthal angles suggested in the "Trento conventions" [33]. To shorten the notation, we introduce the Collins angle $\phi \equiv \phi_{S}+\phi_{h}$. We compute the quantities [2,20]

$$
\begin{gathered}
2 \frac{\int \frac{d x d y}{x y^{2}}(1-y) \sum_{q} e_{q}^{2} h_{1}^{q}(x) H_{1}^{\perp(1 / 2) q}(z)}{\int \frac{d x d y}{x y^{2}}\left(1-y+\frac{y^{2}}{2}\right) \sum_{q} e_{q}^{2} f_{1}^{q}(x) D_{1}^{q}(z)} \approx 2\langle\sin \phi\rangle_{U T}, \quad \text { (36) } \\
2 \frac{\int \frac{d x d y}{x y^{2}}(1-y) z \sum_{q} e_{q}^{2} h_{1}^{q}(x) H_{1}^{\perp(1) q}(z)}{\int \frac{d x d y}{x y^{2}}\left(1-y+\frac{y^{2}}{2}\right) \sum_{q} e_{q}^{2} f_{1}^{q}(x) D_{1}^{q}(z)} \approx 2\left\langle\frac{\left|\vec{P}_{h \perp}\right|}{m_{\pi}} \sin \phi\right\rangle_{U T},
\end{gathered}
$$

where $q$ denotes the quark flavor, $f_{1}^{q}$ is the standard unpolarized parton distribution function (also denoted by $q$ ), and $h_{1}^{q}$ is the transversity distribution function (also denoted by $\delta q$ or $\Delta_{T} q$ ). The equivalence between the expressions on the left-hand side and the experimental asymmetries on the right-hand side (see Ref. [16]) is approximate since power corrections and $\alpha_{S}$ corrections are not taken into account. Moreover, we assumed a full integration over the outgoing hadron's transverse momentum and in Eq. (36) no intrinsic quark transverse momentum in the target is present. ${ }^{2}$

For numerical calculations, we use the nonrelativistic assumption $h_{1}=g_{1}$ (where $g_{1}$ is the helicity distribution function, also denoted by $\Delta q$ ) and the simple parametrization of $g_{1}$ and $f_{1}$ suggested in [36]. In our model, disfavored fragmentation functions vanish, while this is not the case in experiments. Therefore, we focus only on $\pi^{+}$production, where the impact of this limitation of the

\footnotetext{
${ }^{2}$ For a discussion on how results are modified by introducing some initial transverse momentum see Ref. [23].
} 

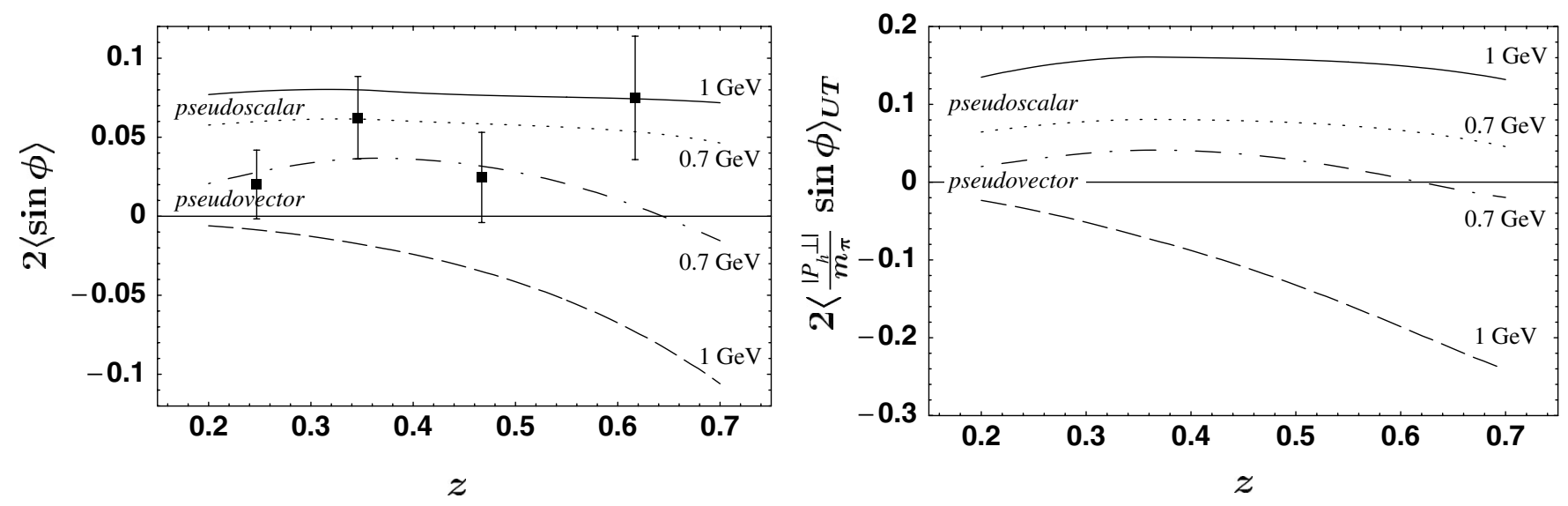

FIG. 13. Azimuthal transverse spin asymmetries $2\langle\sin \phi\rangle_{U T}$ and $2\left\langle\sin \phi\left|\vec{P}_{h \perp}\right| / m_{\pi}\right\rangle_{U T}$ for $\pi^{+}$production at HERMES, assuming $h_{1}=g_{1}$ (see text), for both versions of the pion-quark coupling and for two different values of the parameter $\mu$. Data points for the unweighted asymmetry are from [16].

model should not be very relevant. We apply the kinematical cuts used in the HERMES experimental paper [16].

The results for the asymmetries are plotted in Fig. 13. Published HERMES data [16] are also shown. We want to stress once more some caveats to be taken into account when interpreting these results: (1) the models could be modified by the introduction of form factors, (2) no calculation of the real part of the loop corrections has been made, and disfavored fragmentation functions vanish in this approach, and (3) it is possible that the measured asymmetry receives contributions not included in the standard leading-order approach. Given the size of the experimental errors (only statistical errors are included), it is difficult to discriminate between the models. Present data exclude the pseudovector coupling with a high value of the parameter $\mu$. In general we observe that, making a reasonable choice of parameters, we are not able to describe the data in the framework of the discussed models by using pionic degrees of freedom only.

\section{CONCLUSIONS}

In this paper we reviewed four model calculations of the Collins function for the fragmentation of a quark into a pion, which differ in the type of pion-quark coupling and in the type of one-loop corrections they consider: pseudoscalar pion-quark coupling with pion loops [19] and with gluon loops [21]; pseudovector pion-quark coupling with pion loops [20] and with gluon loops [22]. Even if these models have been already discussed in the literature, we felt the need of presenting revised calculations in order to fix some errors and discuss some details that were not explicitly addressed in the past publications.

For all models, we discussed the shape and parameter dependence of the unpolarized fragmentation function $D_{1}$. The agreement with typical parametrizations of this function is not good for the pseudoscalar coupling. For the pseudovector coupling, the comparison is more encourag- ing. The inclusion of form factors in the pion-quark coupling can however change these results.

In all the above-mentioned approaches disfavored fragmentation functions vanish, while data suggest that the disfavored Collins function could be as big as the favored and have an opposite sign. In principle, disfavored fragmentation functions could be calculated in the framework of the above-mentioned models by considering diagrams with the emission of two pions, one of which goes unobserved.

We computed the Collins function following the definition suggested in the "Trento conventions" [33]. We found an overall sign error in the published calculations of the Collins function in Refs. [19,20,22]. Moreover, we checked that no contribution to the Collins function comes from the pole in the eikonal propagator, in agreement with the general analysis of Ref. [27]. As a consequence, we pointed out that the calculation of the Collins function in Ref. [21] is in our opinion wrong.

For one specific choice of the parameters of the models, we analyzed separately the different diagrams giving rise to a nonzero Collins function and we discussed the size of their contribution to the final result. It turns out that in three out of four cases the contributions of two diagrams roughly cancel each other, so that the Collins function is driven dominantly by one diagram only. This observation holds for different choices of the parameters, too. However, in the case of the pseudovector coupling with gluon loops no dominant diagram can be identified.

The different signs obtained for the Collins function, and, in particular, for the contributions of each diagram separately, lead us to the conclusion that it is not possible to foresee the sign of the Collins function a priori. The fact that more than one diagram with a different structure contributes to the Collins function makes it also difficult, if not impossible, to interpret the resulting effect in terms of simple attractive/repulsive interactions. 
We studied the parameter dependence of the ratio $H_{1}^{\perp(1 / 2)} / D_{1}$ for the two different choices of the pion-quark coupling, summing together pion and gluon loop corrections. Finally, we estimated the single-spin asymmetry for $\pi^{+}$production in semi-inclusive DIS off transversely polarized protons and compared it with available experimental data [16]. The conclusions that can be drawn from the numerical results are somewhat limited. It appears that data cannot be described by using pionic degrees of freedom only. It seems also that data can exclude the pseudovector model with a high value of the parameter $\mu$. All other versions of the models are compatible with the data.

\section{ACKNOWLEDGMENTS}

The work of A. B. has been supported by the Alexander von Humboldt Foundation, the work of A. M. by the Sofia Kovalevskaya Programme of the Alexander von Humboldt Foundation.
[1] J. C. Collins, Nucl. Phys. B396, 161 (1993).

[2] P. J. Mulders and R. D. Tangerman, Nucl. Phys. B461, 197 (1996); B484, 538(E) (1997).

[3] D. Boer and P. J. Mulders, Phys. Rev. D 57, 5780 (1998).

[4] M. Anselmino, M. Boglione, and F. Murgia, Phys. Lett. B 362, 164 (1995).

[5] D. Boer, R. Jakob, and P. J. Mulders, Phys. Lett. B 424, 143 (1998).

[6] A. V. Efremov, Y. I. Ivanshin, O. G. Smirnova, L. G. Tkachev, and R. Y. Zulkarneev, Czech. J. Phys. 49S2, 75 (1999).

[7] A. V. Efremov, K. Goeke, and P. Schweitzer, Phys. Lett. B 522, 37 (2001); 544, 389(E) (2002).

[8] B.-Q. Ma, I. Schmidt, and J. J. Yang, Phys. Rev. D 66, 094001 (2002).

[9] A. V. Efremov, K. Goeke, and P. Schweitzer, Phys. Rev. D 67, 114014 (2003).

[10] M. Anselmino, M. Boglione, U. D’Alesio, E. Leader, and F. Murgia, Phys. Rev. D 71, 014002 (2005).

[11] A. Airapetian et al. (HERMES Collaboration), Phys. Rev. Lett. 84, 4047 (2000).

[12] A. Airapetian et al. (HERMES Collaboration), Phys. Rev. D 64, 097101 (2001).

[13] H. Avakian et al. (CLAS Collaboration), Phys. Rev. D 69, 112004 (2004).

[14] D. L. Adams et al. (FNAL-E704 Collaboration), Phys. Lett. B 264, 462 (1991).

[15] J. Adams et al. (STAR Collaboration), Phys. Rev. Lett. 92, 171801 (2004).

[16] A. Airapetian et al. (HERMES Collaboration), Phys. Rev. Lett. 94, 012002 (2005).

[17] A. V. Efremov, K. Goeke, and P. Schweitzer, hep-ph/ 0412420 .
[18] V. Y. Alexakhin et al. (COMPASS Collaboration), hep-ex/ 0503002.

[19] A. Bacchetta, R. Kundu, A. Metz, and P. J. Mulders, Phys. Lett. B 506, 155 (2001).

[20] A. Bacchetta, R. Kundu, A. Metz, and P. J. Mulders, Phys. Rev. D 65, 094021 (2002).

[21] L. P. Gamberg, G. R. Goldstein, and K. A. Oganessyan, Phys. Rev. D 68, 051501 (2003).

[22] A. Bacchetta, A. Metz, and J.-J. Yang, Phys. Lett. B 574, 225 (2003).

[23] P. Schweitzer and A. Bacchetta, Nucl. Phys. A732, 106 (2004).

[24] L. P. Gamberg, D. S. Hwang, and K. A. Oganessyan, Phys. Lett. B 584, 276 (2004).

[25] L. P. Gamberg, hep-ph/0412367.

[26] D. Boer, P. J. Mulders, and F. Pijlman, Nucl. Phys. B667, 201 (2003).

[27] J. C. Collins and A. Metz, Phys. Rev. Lett. 93, 252001 (2004).

[28] A. Metz, Phys. Lett. B 549, 139 (2002).

[29] X. Ji and F. Yuan, Phys. Lett. B 543, 66 (2002).

[30] A. V. Belitsky, X. Ji, and F. Yuan, Nucl. Phys. B656, 165 (2003).

[31] A. Manohar and H. Georgi, Nucl. Phys. B234, 189 (1984).

[32] S. Kretzer, E. Leader, and E. Christova, Eur. Phys. J. C 22, 269 (2001).

[33] A. Bacchetta, U. D’Alesio, M. Diehl, and C. A. Miller, Phys. Rev. D 70, 117504 (2004).

[34] D. Boer, S. J. Brodsky, and D. S. Hwang, Phys. Rev. D 67, 054003 (2003).

[35] Z. Lu and B.-Q. Ma, Phys. Rev. D 70, 094044 (2004).

[36] S. J. Brodsky, M. Burkardt, and I. Schmidt, Nucl. Phys. B441, 197 (1995). 